\title{
Mecanismos cognitivos en la toma de decisiones en situaciones de incertidumbre. Un estudio con pacientes con demencia tipo Alzheimer
}

\author{
Aurora Moreno y José Ramón Alameda \\ Universidad de Huelva (España)
}

Los pacientes con demencia tipo Alzheimer (DTA) suelen presentar problemas en la toma de decisiones (TD), incluso ya en fase leve. Diversos estudios han analizado el funcionamiento de los mecanismos cognitivos en los procesos de TD, especialmente en situaciones de incertidumbre. Una de estas aproximaciones es la hipótesis del marcador somático a través de la Iowa Gambling Task (IGT) y el índice Gambling (IG). Uno de los problemas es la inespecificidad de los indicadores de la IGT, por ello se han propuesto modelos explicativos para solventar estas carencias, como el Prospective Valence Learning (PVL). En este trabajo, aplicamos la IGT a 10 pacientes y 10 controles. Analizamos los parámetros del PVL: aversión a las pérdidas $(\lambda)$ regla de utilidad $(\alpha)$, recencia (A) y consistencia (c), y la evolución de la tarea en función de las elecciones ventajosas. Nuestros resultados muestran que el rendimiento de los controles es mejor que el de pacientes DTA, aunque en las fases iniciales no hay diferencias, estas se ciñen a los dos bloques finales. En los parámetros del PVL se obtienen diferencias en $\alpha$ y c y en menor medida en $\lambda$. A partir de los parámetros del PVL podemos caracterizar el proceso de toma de decisiones de los pacientes DTA, como sensibles a las pérdidas, más influenciados por las elecciones inmediatas y un nivel de consistencia muy bajo, que indica estrategias de elección aleatoria.

Palabras clave: Toma de decisiones, demencia tipo Alzheimer, programa Cartas, modelo prospect-valence learning.

Cognitive mechanisms underlying risky decision-making. A study of patients with Alzheimer's dementia. Patients with mild dementia of Alzheimer's type (DAT) use to present problems in decision making. Several studies have analyzed the cognitive functions in the process of decision making, especially in situations under ambiguity. One approach is the somatic marker hypothesis from the Iowa Gambling Task (IGT) and the Gambling Index (IG). One problem is the lack of specificity from IGT indicators, for this reason some hypotheses have been proposed in order to solve these deficiencies, i.e. the Prospective Valence Learning (PVL). In this study, we apply the IGT to 10 patients and 10 control subjects. We analyze the PVL parameter: loss aversion parameter $(\lambda)$, shape parameter $(\alpha)$, recency parameter $(A)$, consistency (c) and task development in function of advantageous choices. Our results show that control subjects' performance is better than $\mathrm{DAT}^{\prime}$, nevertheless, in the first stages there are not differences, these appear in the two last blocks. Whit the PVL parameters we obtain differences in $\alpha$ and c, and, to a lesser extent, in $\lambda$. According to PVL parameters, DAT patients can be described as sensible at loss subjects who are more influenced by immediate choice and a very low level of consistence, what implies the use of random choice strategies.

Key words: Decision-making, Alzheimer type dementia, software Cartas, prospect-valence learning model.

Correspondencia: Aurora Moreno Márquez. Facultad de Psicología. Universidad de Huelva. Campus de El Carmen. Avenida de las Fuerzas Armadas, s/n. 21071. Huelva (España). E-mail: aurora.moreno@alu.uhu.es 
Las investigaciones sobre la toma de decisiones se han orientado tradicionalmente al estudio con pacientes con lesión prefrontal ventromedial. Damasio y Bechara han dedicado muchos de sus estudios hacia este ámbito, hallando que los pacientes con lesión cerebral en las estructuras y áreas de la región prefrontal ventromedial, presentan dificultades para tomar decisiones ventajosas y aprender del refuerzo positivo o negativo que se obtiene al tomar la decisión (Damasio, 2006).

Bechara, Damasio, Damasio y Anderson (1994) propusieron la Iowa Gambling Task (IGT) para evaluar la toma de decisiones y como medida del marcador somático. Estos estudios del grupo de Iowa muestran que los resultados de los sujetos controles son mejores que los de los pacientes ventromediales, dado que éstos presentan déficits emocionales. Un marcador somático (Damasio, 1994/2009) es un cambio corporal que refleja un estado emocional, ya sea positivo o negativo, que puede influir en las decisiones tomadas en un momento determinado. Cuando existen diferentes posibilidades de actuación, la corteza prefrontal es capaz de representar, de manera fugaz, los diversos escenarios que pueden derivarse de las distintas decisiones. Las imágenes contienen elementos descriptivos y a su vez, marcadores de las reacciones emocionales que se derivan de cada decisión.

La demencia tipo Alzheimer (DTA) es una enfermedad degenerativa que afecta al cerebro y que se manifiesta por problemas de memoria, de pensamiento y de conducta. En los estadios iniciales de la enfermedad, la apoptosis o muerte neuronal se produce en las áreas frontotemporales y el cuerpo estriado. La alteración moderada del hipocampo y los circuitos límbicos se ve reflejada por la degeneración neurofibrilar y las placas seniles que se sitúan en las regiones etnorrinal y transentorrinal, afectando a las capas profundas (Braak y Braak, 1996). Dado que las áreas frontales y temporales (especialmente circuitos límbicos) son las más afectadas en los estadios iniciales de la DTA, sería de interés conocer cómo actúan las personas que sufren esta demencia en situaciones de toma de decisiones. Los estudios dedicados al estudio de la toma de decisiones en pacientes DTA (Delazer, Sinz, Zamarian y Benke, 2007; Sinz, Zamarian, Benke, Wenning y Delazer, 2008) son aun incipientes, apuntando hacia una conducta inespecífica de decisión y de incapacidad de aprender de las consecuencias.

En la IGT, a los participantes se le presenta cuatro montones de cartas y tienen que seleccionar algún montón sin conocer las posibles consecuencias de la elección. Dos de los montones de cartas ofrecen grandes ganancias pero a la vez, grandes pérdidas a largo plazo (mazos desventajosos); los otros dos, presentan ganancias más moderadas y pequeñas pérdidas (mazos ventajosos). Damasio (1994/2009) sugiere que las decisiones de los sujetos sanos están mediadas por el marcador somático, los cuales presentan una tendencia creciente a elegir los mazos ventajosos.

Para el análisis de los resultados de la tarea, el grupo de Busemeyer y Stout proponen en 2002 el modelo matemático Expectancy Valence Learning (EVL), que es 
mejorado posteriormente con el modelo Prospect Valence Learning (PVL), de Ahn, Busemeyer, Wagenmakers y Stout (2008). PVL redefine las ecuaciones para establecer el valor de la carta y la consistencia entre elecciones y expectativas, lo que proporciona una mayor potencia explicativa al modelo PVL (Fridberg, Queller, Ahn, Kim, Bishara, Busemeyer, Porrino y Stout, 2010). Según este modelo, los mecanismos atencionales atribuyen el valor ponderado a una carta, que puede ser de ganancia, de pérdida o ambas. Este valor es representado a través de una función matemática no lineal. El modelo parte de cuatro ecuaciones que permiten obtener el valor de la carta, la creación de expectativas, la probabilidad de elección de cada mazo y la consistencia entre elecciones y expectativas. Las ecuaciones concretas son:

1.- Obtención del valor de la carta:

$\mathrm{u}(\mathrm{t})=\left|\begin{array}{cc}\chi(\mathrm{t}) \alpha & \text { si } \chi(\mathrm{t}) \geq 0 \\ -\lambda\{\chi(\mathrm{t}) \alpha\} & \text { si } \chi(\mathrm{t})<0\end{array}\right|$ donde $\left|\begin{array}{lc}\alpha & \text { Regla de utilidad } \\ \lambda & \text { aversión a las pérdidas }\end{array}\right|$

2.- Creación de la expectativa $E$ para el mazo j en la jugada $t$

$\mathrm{Ej}=\mathrm{E}_{\mathrm{j}}(\mathrm{t}-1)+\mathrm{A} \cdot \delta_{\mathrm{j}}(\mathrm{t}) \cdot\left[\mathrm{u}(\mathrm{t}) \mathrm{E}_{\mathrm{j}}(\mathrm{t}-1)\right]$ donde $\mathrm{A}=$ Recencia

3.- Probabilidad de elección del mazo j

$\operatorname{Pr}[D(t+1)=j]=\frac{e^{\theta(t) E_{j}(t)}}{\sum_{j=1}^{4} e^{\theta(t) E_{j}(t)}}$

4.- Consistencia entre elecciones y expectativas

$\theta(t)=3^{c}-1$ donde $\mathrm{c}=$ Consistencia

Para el desarrollo de tres de estas cuatro ecuaciones es necesario el cálculo de cuatro parámetros, $\alpha$ y $\lambda$ para la primera ecuación: A para la segunda y c para la cuarta. El significado e interpretación de estos parámetros los vemos a continuación:

Aversión a las pérdidas $(\lambda)$. En relación a la sensibilidad del sujeto a las pérdidas comparadas con las ganancias. Este parámetro explica el fenómeno de preferencia del mazo B, que aunque desventajoso, la frecuencia de pérdidas es reducida. Los valores de $\lambda$ oscilan entre 0 y 1 . Valores de 0 indican que no existe sensibilidad a las pérdidas, que los sujetos están experimentando los acontecimientos como neutros. La $\lambda=1$ en relación a que las ganancias y las pérdidas tienen el mismo impacto. Valores mayores que uno, reflejan aversión a las pérdidas, dado que éstas tienen un impacto mayor que las ganancias.

Regla de utilidad $(\alpha)$, tiene un valor comprendido entre 0 y $1(0<\alpha<1)$. Valores próximos a 0 indican utilidad subjetiva en las elecciones, la cual otorga mayor importancia al número de ganancias y pérdidas, más que al valor de los resultados (las cantidades en valor monetario obtenidas). Valores próximos a 1, indican que la utilidad subjetiva se relaciona directamente con el valor de los resultados.

Recencia (A), sobre la experiencia reciente y la creación de expectativas. Representa el peso que le da el sujeto a las experiencias previas frente a la última elección que ha llevado a cabo. Es un índice de la tasa de aprendizaje, en un rango de 0 a 
1, donde los valores próximos a 1 indican que la carta más reciente tiene una gran influencia para la próxima elección, olvidando el resto de elecciones pasadas. Los valores próximos a 0 manifiestan que las elecciones pasadas tienen un gran peso para elegir en la actualidad, así las expectativas sobre un mazo están influenciadas por el aprendizaje previo, el olvido es más gradual, a la vez que las expectativas se actualizan con cada carta nueva seleccionada.

Consistencia (c) es un indicador de la correspondencia entre las selecciones del participante y las expectativas a medida que progresa la tarea, se trata de la probabilidad de elección de un mazo. Los valores oscilan entre 0 y 5: un valor alto de c explica que las opciones de elección son deterministas $\mathrm{y}$, un valor bajo de c, poca coherencia, siendo las elecciones aleatorias.

En definitiva, para realizar correctamente la IGT el participante debe aprender a seleccionar los mazos ventajosos a partir del balance de ganancias-pérdidas que experimentan a través de la tarea. Por lo tanto, la IGT incorpora tanto mecanismos cognitivos (p.e., aprendizaje y memoria) como procesos motivacionales (p.e., la capacidad de respuesta ante ganancias-pérdidas) asociadas con la anticipación de los resultados a través de las experiencias previas.

Decisiones como tomar o no tomar drogas, hacer o no hacer una determinada compra o inversión, etc., también guardan relación con los procesos relacionados con el aprendizaje de experiencias previas, y las recompensas percibidas (p.e., el placer) y castigos (p.e., financieros, problemas interpersonales, legales) asociados con las decisiones tomadas.

Los modelos computacionales cognitivos como el PVL permiten desentrañar los procesos que contribuyen al rendimiento en la IGT y para identificar específicamente a aquellos que pueden explicar el comportamiento más general de una persona o grupo en la tarea (Busemeyer y Stout, 2002). Por ello en este trabajo vamos a aplicar los parámetros del PVL a un grupo de pacientes con demencia tipo Alzheimer frente a un grupo control. En la línea de trabajos previos como los del grupo de Stout, esperamos encontrar diferencias significativas en los cuatro parámetros en ambos grupos, lo que nos permitirá obtener una medida más precisa del proceso de toma de decisiones.

\section{MÉTODO}

\section{Participantes}

Han participado en este trabajo 10 pacientes con DTA y 10 sujetos controles. Las personas con DTA han sido diagnosticadas por el Servicio Andaluz de Salud y son usuarios del Centro de Día "Rocío Sánchez" de la AFA de Huelva: 5 hombres y 5 mujeres, situados en estadio 3-4 de la Escala de Deterioro Global (GDS) de Reisberg (deterioro leve a moderado), con deterioro cognitivo leve y edades entre 60-80 años, similar nivel de estudios y sin tratamiento psicofarmacológico. La edad promedio fue de 
69,1 para los sujetos experimentales y de 69,5 para los controles, con una $t_{(1,18)}=-0,124$ $(\mathrm{p}=0,903)$. Las diferencias sobre los años de escolarización y la edad son reducidas y no significativas.

\section{Instrumentos}

Batería de cribado en demencias. El grado de deterioro cognitivo se evaluó a través del Miniexamen Cognoscitivo de Lobo (MEC), versión española del Minimental Test. Se tuvieron en cuenta los ítems de orientación temporal, espacial, de memoria y recuerdo, atención y cálculo, praxias y lenguaje. Utilizamos además el Test del Reloj (Test del Reloj a la Orden y Test del Reloj Copia, de Kaplan y Goodlass; la fluidez verbal a través de la Subescala Animales (durante un minuto) del Test de Fluidez Verbal de Isaacs.

Programa "Cartas", versión informática de la IGT. En la IGT el sujeto debe elegir entre cuatro montones de cartas, las cuales implican pérdidas o ganancias monetarias simbólicas en cada ensayo. Los primeros montones (A y B) implican grandes ganancias, pero a la vez, cuantiosas pérdidas y, los dos últimos (C y D) implican ganancias en menor cuantía, y poca pérdida, por tanto conducen a ganar. Con A se ganan $100 €$ en cada elección, y pérdidas variables de 150 a $350 €$ en 5 de cada 10 jugadas. Con $\mathrm{B}$ se ganan $100 €$ en cada jugada y tiene un única pérdida de $1250 €$ por cada 10 jugadas. En $C$, se ganan $50 €$ en cada elección y se pierden de 25 a $75 €$ en cinco de cada 10 jugadas. En D, las ganancias son de $50 €$ y una única pérdida de $250 €$ por cada 10 jugadas. Haciendo balance, con A y B por cada 10 elecciones se pierden $250 €$, mientras que con $\mathrm{C}$ y $\mathrm{D}$, se ganan $250 €$ por cada ciclo de 10 jugadas. El objetivo del juego es conseguir el mejor saldo positivo.

\section{RESULTADOS}

Tabla 1. Resultados de las pruebas de cribado del deterioro cognitivo

\begin{tabular}{|c|c|c|c|c|}
\hline & \multicolumn{2}{|c|}{ M. (D.T) } & \multirow{2}{*}{ Valor Sig. ${ }^{a}$} & \multirow[b]{2}{*}{$t(\mathrm{gl})$} \\
\hline & Sujetos DTA & Controles & & \\
\hline $\operatorname{MEC}(\mathrm{x} / 35)^{\mathrm{b}}$ & $24,7(3,52)$ & $34,3(0,9)$ & 0,000 & $-8,307(1,18)$ \\
\hline Orientación MEC $(\mathrm{x} / 10)^{\mathrm{c}}$ & $6,6(2,6)$ & $9,9(0,316)$ & 0,003 & $-3,935(1,18)$ \\
\hline Memoria Fijación MEC $(\mathrm{x} / 3)^{\mathrm{c}}$ & $3(0,00)$ & $3(0,00)^{\mathrm{d}}$ & & \\
\hline Cálculo MEC $(\mathrm{x} / 8)^{\mathrm{c}}$ & $5(1,82)$ & $8(0,0)$ & $\overline{0,01}$ & $-5,196(1,18)$ \\
\hline Memoria Repetición MEC (x/3) ${ }^{\mathrm{c}}$ & $0,2(0,42)$ & $2,4(0,84)$ & 0,00 & $-7,379(1,18)$ \\
\hline Lenguaje y Construcción MEC $(\mathrm{x} / 11)^{\mathrm{c}}$ & $10,2(0,91)$ & $11(0,00)$ & 0,022 & $-2,753(1,18)$ \\
\hline 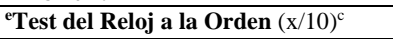 & $7,4(2,5)$ & $10(0,00)$ & 0,010 & $-3,228(1,18)$ \\
\hline${ }^{\mathrm{f} T e s t}$ del Reloj Copia $(\mathrm{x} / 10)^{\mathrm{c}}$ & $8,9(1,4)$ & $10(0,00)$ & 0,040 & $-2,400(1,18)$ \\
\hline 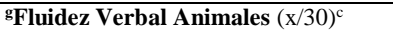 & $9,2(2,2)$ & $35,4(3,9)$ & 0,00 & $-18,324(1,8)$ \\
\hline
\end{tabular}

$\mathrm{M}=$ media; D.T.=desviación típica. ${ }^{\text {a }}$ Valor significación (bilateral) para Prueba T para la igualdad de medias. Gl=Grados de libertad. ${ }^{\text {b }}$ Punto de corte $=>30$, puntuación límite de inicio de deterioro cognitivo. 23-24 (deterioro cognitivo). ${ }^{\mathrm{c}} \mathrm{x}=$ Puntuación obtenida en el test/puntuación máxima que se puede obtener. ${ }^{\mathrm{d}}$ (No puede calcularse T puesto que las desviaciones típicas de ambos grupos son 0). ${ }^{\mathrm{e}}$ Punto de corte $=6 .{ }^{\mathrm{f}}$ Punto de $\operatorname{corte}=8$. ${ }^{\mathrm{g}}$ Punto de corte $=10-14$ palabras/minuto. 


\section{Deterioro cognitivo}

A fin de constatar que los pacientes y controles se diferenciaban efectivamente en la manifestación y ausencia de deterioro cognitivo, se tomaron datos del nivel de deterioro, que fueron significativamente diferentes para ambos grupos (ver Tabla 1).

Parámetros modelo PVL

Los datos obtenidos de los parámetros del modelo PVL han sido los siguientes (Tabla 2).

Tabla 2. Descriptivos de los parámetros del modelo PVL

\begin{tabular}{lcccccc}
\hline & \multicolumn{2}{c}{ DTA } & \multicolumn{7}{c}{ Controles } \\
\cline { 2 - 7 } & $M$ & $D T$ & $M$ & $D T$ & $t_{(1-18)}$ & $p$ \\
\hline Recenc &, 488 &, 437 &, 368 &, 262 &, 747 &, 467 \\
\hline Alpha &, 751 &, 358 &, 234 &, 410 & 3,033 &, 008 \\
\hline Consist &, 098 &, 053 &, 567 &, 357 & 4.121 &, 002 \\
\hline Lambda & 3,286 & 1,533 & 2,245 & 2,039 & 1,828 &, 084 \\
\hline Elec. Vent. &, 489 &, 040 &, 560 &, 051 & 3,460 &, 003 \\
\hline
\end{tabular}

Como podemos apreciar, las diferencias significativas se dan sólo en los parámetros Alpha y consistencia, en el caso de Lambda, se aproxima a la significatividad. También hay diferencias en cuanto al porcentaje de elecciones de los mazos ventajosos, ya que como podemos apreciar en la tabla, los pacientes DTA, realizan en el cómputo total de la prueba elecciones ventajosas por debajo del $50 \%$ (Ver Figura 1).

Figura 1. Representación gráfica de los parámetros del modelo PVL

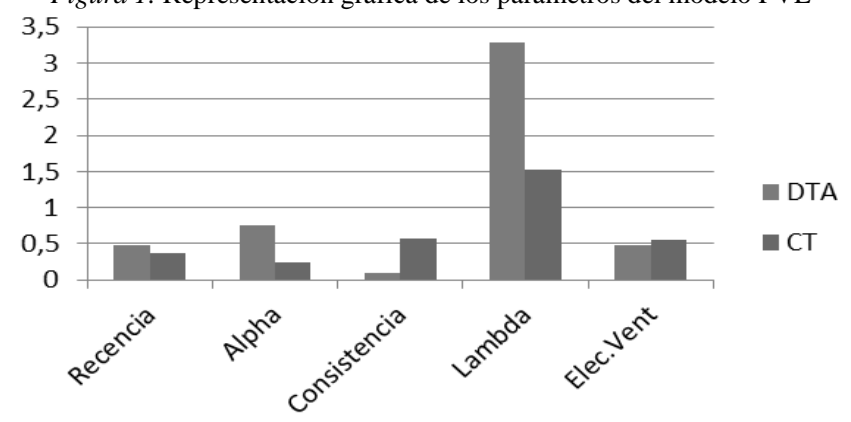

Siguiendo el orden en los parámetros del modelo PVL, podemos apreciar que en el establecimiento del valor de la carta, no hay diferencias en cuanto a la aversión a las pérdidas (Lambda), y en ambos casos podríamos afirmar que hay aversión a las pérdidas, ya que el valor de Lambda es superior a 1 . Si hay diferencias en cuanto a Alpha, o forma, en este caso los valores de los pacientes están más próximos a 1 por lo 
que su conducta de elección está más asociada a los montantes de las pérdidas y las ganancias, mientras que los controles están influenciados más por la frecuencia de las mismas que por el monto total. En cuanto a la creación de expectativas, medida a partir del parámetro recencia (A) no hay diferencias significativas en ambos grupos y las puntuaciones podrían ser consideradas intermedias, por lo que hay cierto equilibrio entre el peso dado a las expectativas de cada mazo y la elección reciente. Por último, el parámetro consistencia es muy bajo en los pacientes DTA, mientras que en los controles está por encima del 50\%, lo que denota una ejecución aleatoria en el caso de los pacientes. Esta ejecución aleatoria condiciona en parte los datos obtenidos en los otros parámetros, ya que son el resultado de elecciones al azar, más que de estrategias.

En el análisis de las elecciones ventajosas por bloques obtenemos los siguientes resultados (Tabla 3 y Figura 2).

Tabla 3. Porcentaje de elecciones ventajosas en los 5 bloques

\begin{tabular}{ccccccc}
\hline & \multicolumn{2}{c}{ DTA } & \multicolumn{2}{c}{ Controles } & $p$ \\
\cline { 2 - 7 } & $M$ & $D T$ & $M$ & $D T$ & $t_{(1-18)}$ &, 618 \\
\hline B1 &, 455 &, 109 &, 430 &, 111 &, 508 &, 327 \\
\hline B2 &, 470 &, 092 &, 520 &, 127 & 1,007 &, 157 \\
\hline B3 &, 495 &, 093 &, 565 &, 118 & 1,476 &, 045 \\
\hline B4 &, 490 &, 094 &, 630 &, 183 & 2,155 &, 038 \\
\hline B5 &, 535 &, 135 &, 655 &, 101 & 2,243 & \\
\hline
\end{tabular}

Figura 2. Curva de aprendizaje

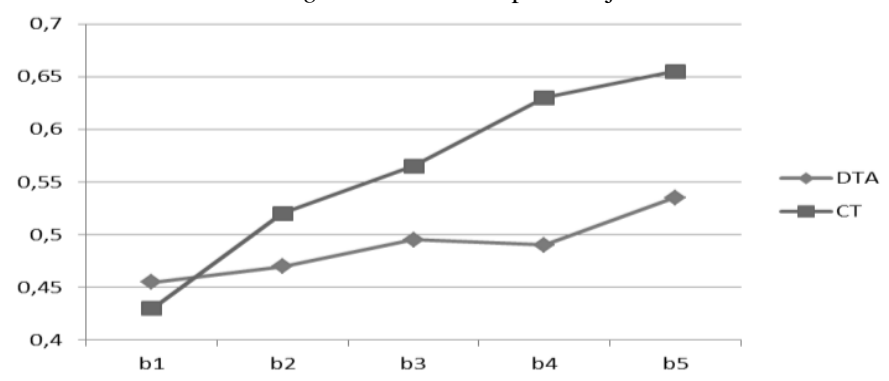

Como podemos apreciar hay una clara tendencia ascendente en los controles, mientras que en los pacientes DTA, tan sólo en el último bloque se nota una ejecución mejor. En los bloques iniciales no hay diferencias significativas, estas son evidentes en los dos últimos bloques, lo que pone de manifiesto como los controles van mejorando a lo largo de la tarea y los pacientes DTA lo hacen en menor medida.

Analizando estos resultados a través del correspondiente análisis de varianza de medidas repetidas obtenemos que hay diferencias significativas para el factor principal Bloque $\left(F_{(4-15)}=3,164 ; p=, 045\right)$ Sin embargo, no se obtienen diferencias 
significativas en el factor intersujetos $\left(F_{(4-15)}=, 989 ; p=, 443\right)$, si analizamos los datos de forma separada para ambos grupos, obtenemos diferencias significativas para los controles $\left(F_{(4-6)}=5,037 ; p=, 040\right)$, pero no para los pacientes DTA $\left(F_{(4-6)}=, 291 ; p=, 874\right)$. Estos nos muestra que mientras que sí podemos hablar del aprendizaje de la tarea en los controles, la mejora observada en el último bloque en los pacientes DTA no se ve confirmada estadísticamente. Mediante el contraste de Bonferroni, observamos que no hay diferencias ente el nivel de ejecución de ningún bloque en los pacientes DTA, mientras que en los controles las diferencias se observan entre el primer bloque y el cuarto $(p=, 039)$ y el primero y el quinto $(p=, 011)$.

\section{Deterioro cognitivo y parámetros $P V L$}

Llevamos a cabo correlaciones entre los resultados de las pruebas del deterioro cognitivo y los parámetros PVL, obteniendo correlaciones significativas entre el parámetro $\alpha$ (forma) y las subpruebas de orientación, memoria de repetición y Fluidez Verbal. Con el parámetro c (consistencia) se obtuvieron correlaciones significativas con orientación, cálculo, memoria de repetición y fluidez verbal (ver tablas 4 y 5).

Tabla 4. Correlaciones $\alpha$ y deterioro cognitivo

\begin{tabular}{lccc}
\hline & $\begin{array}{c}\text { Coeficiente de } \\
\text { correlación }\end{array}$ & Significación & $\begin{array}{c}\text { Coeficiente de } \\
\text { determinación }\end{array}$ \\
\hline MEC &, 504 &, 024 &, 254 \\
\hline Orientación &, 504 &, 023 &, 254 \\
\hline Cálculo &, 191 &, 419 &, 036 \\
\hline Memoria de repetición &, 812 &, 000 &, 659 \\
\hline Lenguaje y construcción &, 376 &, 102 &, 141 \\
\hline Test Reloj a la Orden &, 185 &, 435 &, 034 \\
\hline Test Reloj Copia &, 112 &, 637 &, 012 \\
\hline FV animales &, 506 &, 023 &, 256 \\
\hline
\end{tabular}

Tabla 5. Correlaciones c y deterioro cognitivo

\begin{tabular}{lccc}
\hline & $\begin{array}{c}\text { Coeficiente de } \\
\text { correlación }\end{array}$ & Significación & $\begin{array}{c}\text { Coeficiente de } \\
\text { determinación }\end{array}$ \\
\hline MEC &, 67 &, 001 &, 449 \\
\hline Orientación &, 487 &, 29 &, 023 \\
\hline Cálculo &, 539 &, 014 &, 290 \\
\hline Memoria de repetición &, 807 &, 000 &, 651 \\
\hline Lenguaje y construcción &, 338 &, 145 &, 114 \\
\hline Test Reloj a la Orden &, 426 &, 061 &, 181 \\
\hline Test Reloj Copia &, 350 &, 130 &, 122 \\
\hline FV animales &, 623 &, 003 &, 388 \\
\hline
\end{tabular}

\section{DISCUSIÓN}

Los modelos computacionales cognitivos permiten desentrañar los procesos que contribuyen al rendimiento en la IGT y para identificar específicamente a aquellos que pueden explicar el comportamiento más general de una persona o grupo en la tarea 
(Busemeyer y Stout, 2002). Se ha empleado el modelo PVL para el análisis de la ejecución en la prueba Cartas de nuestros pacientes. Se han hallado resultados destacables en relación a que los pacientes realizan en la mayoría de ocasiones elecciones desventajosas que, en ocasiones, experimentan aversión a las pérdidas. El modelo detectó cierto equilibrio entre el peso dado a las expectativas de cada mazo y la elección reciente; no obstante, se vio como las elecciones que hacían eran aleatorias, elegían al azar cualquier carta. Tampoco se puede afirmar que aprendieran de la prueba, pues los estadísticos que se obtienen no son significativos, no obstante, la curva nos mostró que a final de la prueba experimentan un incremento en la elección de mazos ventajosos. A partir de lo observado, podríamos afirmar que en estadios iniciales de la DTA, las personas presentan dificultades para tomar decisiones acertadas en situaciones de incertidumbre. Todavía son escasos los trabajos que aplican el PVL a los pacientes con DTA, con otras poblaciones clínicas muestran claras diferencias en todos los parámetros con respecto a grupos controles (Busemeyer y Stout, 2002). En este sentido nuestro trabajo no coincide exactamente, ya que sólo obtenemos diferencias significativas en dos de cuatro, regla de utilidad y consistencia.

Acerca de las correlaciones de los parámetros y las pruebas del deterioro, destacar el papel de la consistencia. Se ha visto que nuestros pacientes realizan elecciones aleatorias, y esto se ha relacionado con las subpuntuaciones del MEC y otras pruebas del deterioro. Apuntan estas correlaciones a que las personas que obtienen peores puntuaciones en cálculo, recuerdan peor o tienen dificultades para evocar palabras, son las que realizan elecciones más incoherentes.

Con todo, habría que tomar cautela en las situaciones de la vida diaria en las que estas personas se encuentran en circunstancias de elección, como las compras en el mercado, decisiones sobre el testamento o su legado, tomar o no la medicación, etc.

En este trabajo hemos tratado de hacer una descripción del proceso de toma de decisión a través del análisis de parámetros que se asocian con una buena o mala elección, como han sido la aversión a las pérdidas, la creación de expectativas, la forma o consistencia de la respuesta, frente a otras medidas más imprecisas que se utilizan en este tipo de trabajo (en relación al Índice Gambling, que se centra en la tendencia o no al riesgo). Entre los aspectos a mejorar para futuras investigaciones, destacar la ampliación de la muestra o el empleo de otras pruebas que evalúen la toma de decisiones y el deterioro cognitivo.

\section{REFERENCIAS}

Ahn, W.Y., Busemeyer, J.R., Wagenmakers, E.J. y Stout, J.C. (2008). Comparison of decision learning models using the generalization criterion method. Cognitive Science, 32, 13761402 . 
Bechara, A., Damasio, A.R., Damasio, H. y Anderson S.W. (1994). Insensitivity to future consecuences following damage to human prefrontal cortex. Cognition, 50, 7-15.

Braak, H. y Braak, E. (1996). Evolution of neuropathology of Alzheimer's disease. Acta Neurol Scan, 165, 3-12.

Busemeyer, J.R., y Stout, J.C. (2002). A contribution of cognitive decision models to clinical assessment: Decomposing performance on the Bechara gambling task. Psychological Assessment, 14(3), 253-262.

Damasio, A.R. (1994/2009). El error de Descartes. Barcelona: Drakontos Bolsillo.

Damasio, A.R. (2006). The somatic marker hypothesis and the possible functions of the prefrontal cortex. Philosophical Transactions of the Royal Society of London. Series B, Biological Sciences, 351, 1413-1420.

Delazer, M., Sinz, H., Zamarian, L. y Benke T. (2007). Decision-making with explicit and stable rules in mild Alzheimer's disease. Neuropsychologia, 45, 1632-1641.

Fridberg, D.J., Queller, S., Ahna, W., Kima, W., Anthony J., Bichara, A.J., Busemeyera, J.R., Porrino, L. y Stout, J.C. (2010). Cognitive mechanisms underlying risky decisionmaking in chronic cannabis users. Journal of Mathematical Psychology, 54, 28-38.

Sinz, H., Zamarian, L., Benke, T., Wenning, G.K. y Delazer, M. (2008). Impact of ambiguity and risk on decision making in mild Alzheimer's disease. Neuropsychologia, 46, 2043-2055.

Recibido: 5 de mayo de 2012

Recepción Modificaciones: 5 de junio de 2012

Aceptado: 15 de junio de 2012 